\title{
Protocol Integration for Intelligent Monitoring Applications in Wireless Sensor Networks
}

\author{
Antonio M. Ortiz, Fernando Royo, Teresa Olivares, Luis Orozco-Barbosa, \\ José Carlos Castillo and Antonio Fernández-Caballero \\ Albacete Research Institute of Informatics \\ University of Castilla-La Mancha, 02071 Albacete, Spain \\ \{amanuel, froyo, teresa, lorozco, josecarlos, caballer\}@dsi.uclm.es
}

\begin{abstract}
Integration of algorithms and protocols from different layers will make possible the deployment of large-scale wireless sensor networks which are the basis of interesting monitoring applications. The growing number of nodes that comprise within these networks requires a correct organization and efficient node synchronization to ensure data reliability. In this study, we focus on the integration of fuzzy-logic based routing with a TDMA MAC protocol. By considering the experimental results of them working separately, we have integrated them to work together. The use of a rapid configuration and efficient slot assignment from the MAC protocol, and the accuracy of the logical tree created using fuzzy logic, allows to have a network in which nodes are both organized and synchronized, while load balancing extends network lifetime.
\end{abstract}

Keywords: Wireless sensor networks, Fuzzy-logic, MAC, Routing, Cross-Layer Integration.

\section{Introduction}

Wireless sensor networks comprise a large number of sensor devices that communicate with each other via wireless channels, with limitations of energy and computing capabilities. The efficient and robust realization of such large, highlydynamic and complex networking environments is a challenging algorithmic and technological task [1].

Our research takes a cross-layer-based approach, working with the components of an emerging general-purpose sensor networking infrastructure. Networking is important because it provides the glue that allows individual nodes to collaborate. Radio communication is the major consumer of energy in small sensor nodes. Thus, the optimization of networking protocols can greatly extend the lifetime of the sensor network as a whole [2].

This paper focuses on both the link and network layers. The former layer is approached by considering a media-access control (MAC) protocol, and topology control and a routing approach are discussed for the latter. The MAC layer must manage the communication channel available for the node, and so must avoid collisions and errors in communication. 
Organizing a network, composed in many cases of a high number of lowresourced nodes, is a difficult task since the algorithms and methods have to save as much energy as possible while offering good performance. Power-saving has been the main driving force behind the development of several protocols that have recently been introduced. In this context, perhaps the greatest energy savings are achieved by protocols whose communications are based on time division multiple access (TDMA) and synchronization. However, synchronous communications require the organization of the network nodes in an efficient structure such as a logical tree. This structure can be used to achieve both, collision-free communications and minimum end-to-end latency. The network layer is responsible for the dynamic-routing tree creation and topology control using a novel and interesting routing algorithm.

These kinds of algorithms take into account important node features such as battery level, number of hops to the sink or link quality; note that if this information is not considered, problems in the network may occur (interrupted paths, packet loss or isolated nodes, among others).

Our work integrates the SA-MAC protocol [3], a simple but effective collision resolution protocol as a means to set-up the slot allocations of TDMA protocols, with NORIA (Network rOle-based Routing Intelligent Algorithm) [4], a novel routing algorithm for wireless sensor networks. In this paper, we mainly focus on how SA-MAC and NORIA work together in dense networks in order to optimize the protocols that control the behaviour of network nodes in real applications. In our group, we have developed a multisensory indoor monitoring application, oriented to energy management in intelligent buildings, where we have encountered a number of problems related to information loss and packet collisions. Due to this, we have decided to optimize network and medium access protocols and integrate them together in order to improve the overall performance.

The rest of this paper is organized as follows: Section 2 summarizes some related work. The synchronized medium access protocol, SA-MAC is detailed in Section 3. Fuzzy logic principles are presented in Section 4, and serve as a basis to the intelligent routing approach detailed in Section 5. Section 6 presents the integration of both approaches and details the experiments and obtained results. Finally, Section 7 gives some concluding remarks.

\section{Previous work}

TDMA protocols avoid some important problems such us idle listening, collisions, overhearing and overhead due to control packets. In addition, when an efficient global synchronization mechanism is available, the topology is fairly stable and TDMA protocols are usually the best option for efficient data communications in WSN [5]. A few relevant MAC protocols that make use of TDMA channel sharing are worth mentioning in [3]. SA-MAC protocol solves this setup phase successfully and performs a second phase of synchronization and data transmission. Also, it is strongly recommended for a MAC protocol to be dis- 


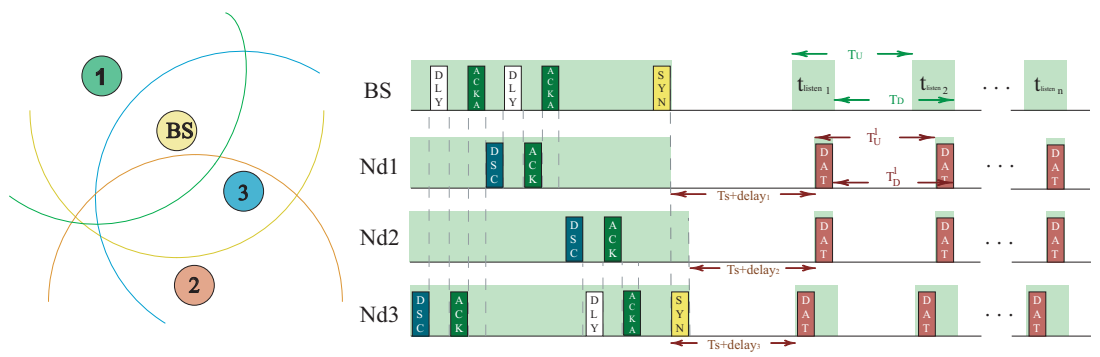

Fig. 1. SA-MAC timing diagram.

tributed and self-organized to support topological changes, since these features are essential to ensure the efficient scalability of a wireless sensor network [6]. In order to do this, we integrate SA-MAC with NORIA, which will create the logical tree in an optimal and intelligent manner, taking into account the differences between nodes and always choosing the most adequate.

In [7] and [8] different routing and self-organizing techniques for WSN are described. These techniques serve as a basis for future routing algorithms which are intended to make the network more efficient. After all, we have came up with the idea of using artificial intelligence techniques to help the decision-making process in order to get more efficient algorithms. Artificial intelligence techniques reinforce the efficiency and the performance of routing algorithms, by combining data from nodes and their interactions in order to make decisions to improve global network performance. Decisions related to information transmission from a source to the sink are one of the most important aspects in sensor networks. Our approach tries to show how artificial intelligence techniques, specifically fuzzy logic, can help these decision-making processes to improve efficiency and to extend network lifetime. We are very interested in studies combining fuzzy rules with networking. In [9] the authors propose two asynchronous MAC protocols, they make a complicated schedule interval and they design a rescheduling fuzzy logic system to monitor the influence of accumulative clock-drifts, the variance of traffic strength and service capability on communications. In their experiments, they increase the number of nodes within a cluster from 5 to 30 and exclude the factors coming from physical layer and network layer to simplify the analysis. Another interesting approach is taken in [10], where the authors propose FuzzyMAC, a CSMA/CA-based MAC protocol that utilizes two separate fuzzy logic controllers to optimize both the MAC parameters and a sleeping schedule duty-cycle. The experiments only show results for 50 nodes and use a proprietary simulator. 


\section{Synchronized MAC}

Power saving has been the main driving force behind the development of several protocols that have been recently appeared in the literature (see [11] for a recent survey). In this context, the largest energy savings are achieved by protocols whose communications are based on time-division multiple access (TDMA) and synchronization.

\subsection{SA-MAC Protocol overview}

The main aim of the SA-MAC protocol is to synchronize the ON/OFF periods of senders and receivers. In the following, the protocol operation will be described by considering a network consisting of a sink node responsible for gathering all the data sensed by all the other nodes. Some of the other nodes may have, when required, to act as relays enabling the forwarding of the collected data to the sink station. The operation of SA-MAC is divided into two phases: 1) the set-up phase and, 2) the synchronization and data transmission phase. In this paper we will briefly describe both of these. Other aspects of the protocol operation can be consulted in [3]. During the set-up phase of the SA-MAC protocol the network nodes exchange four types of packets, namely discovery packets (DSC), delay packets (DLY) and acknowledgement packets (ACK and ACKASOC). In the simplest scenario the set-up phase starts when the base station (e.g., the sink node) announces its presence as a father node so that all other nodes can start trying to establish a father-and-child relation. Thus, all nodes that become aware of the presence of the base station start to broadcast discovery packets (DSC). Upon receiving a DSC packet, the base station sends a DLY packet to the corresponding node. The delay packet indicates the time slot that is assigned for transmissions from the sensor node to the base station. The node acknowledges the DLY packet with an acknowledgement packet, ACK, and this acknowledgement packet will be replied to another one from the parent node named ACKASOC. In this way, the sensor node finishes its association to the base station and then it may become a father node for other nodes.

In order to illustrate the operation of the SA-MAC protocol in a more complex scenario, consider a set of nodes consisting of a base station (BS) and nodes $\mathrm{Nd} 1, \mathrm{Nd} 2$ and $\mathrm{Nd} 3$. Let us assume that $\mathrm{Nd} 1$ and $\mathrm{Nd} 3$ are located within the coverage area of the BS and that Nd2 is located within the coverage area Nd3, but out of reach of the BS. Once the BS announces its presence, nodes Nd1 and Nd3 can start sending DSC packets and collisions may occur at this time. Let us assume that Nd3 resolves the collision and sends its DSC packet to the basestation and establishes a father-and-child relation with the BS. At this point in time, Nd2 chooses the Nd3 node as father because it is the nearest one to the BS and if $\mathrm{Nd} 2$ detects that the channel is free, it can start to send its DSC packets. Nd1 detects the end of the association between Nd3 and BS and it sends its DSC, establishing a father-and-child relation too. Once all the nodes in the first routing level have been associated, nodes from the second one can start to send their DSC packets. When choosing the best father, the nodes of a lower level 
listen the channel to catch the information from the ACK and ACKASOC packets from the nodes of the upper level. These packets include information about the number of hops to the BS; actually, only this parameter has been taken into consideration in the initial design. Once the network has been created, the BS waits for a period of time, during which no new nodes are detected. The BS node will then transmit a SYN packet indicating the beginning of the data transmission phase. This process starts at the base station and a SYN packet is propagated among successive levels. Figure 1 shows the sequence of actions described for this scenario.

During the second phase, the nodes will turn ON and OFF, thus completing a duty cycle. This is to say, the parent node will wake up and each node will transmit within the slot allocated during the set-up phase. In this way, we limit the overhead due to the transient period every time the radio interface is turned on.

\section{Fuzzy Logic}

Fuzzy logic is a decision system approach which works similarly to human control logic. It provides a simple way to arrive at a definite conclusion based upon imprecise, vague or ambiguous input information. Furthermore, only a few data samples are required in order to extract the final accurate result. Besides, fuzzy logic is a handy technique since it uses human language to describe inputs and outputs [12]. All these features make fuzzy logic appropriate for the parent decision process in wireless sensor networks.

One of the frequently-used fuzzy-logic inference methods is Mamdani [13], which consists of four phases: fuzzification, rule evaluation, combination or aggregation of rules, and defuzzification. The input of a Mamdani fuzzy-logic system is usually a crisp value. To allow this value to be processed by the system, it has to be converted to natural language, that is, fuzzified. In this way, the fuzzifier takes numeric values and converts them into fuzzy values which can be processed by the inference system. These fuzzy values represent the membership values of the input variables to the fuzzy sets. Once values have been fuzzified, the inference system processes the fuzzy rules to get a fuzzy output. The third step in the Mamdani inference method is the aggregation of all outputs, where the outputs of each rule are combined to form a new fuzzy set. Finally, in the defuzzification process, the new aggregated fuzzy set is converted to a number. In summary, fuzzy logic is an efficient method for making decisions and it also has the advantage of working with natural language and require low computational capabilities.

\section{$5 \quad$ Network routing}

Routing is a key element in the operation of wireless sensor networks. Paths from any node in the network to the base station must be defined in order to get efficient network communication. With the use of fuzzy-logic, we expect to 
get successful results in terms of network organization time and number of hops from any node to the base station, always choosing the best parent in terms of energy and number of hops to the sink.

\subsection{Fuzzy-logic-based routing}

NORIA is an algorithm capable of route data in a network by creating a communication tree. The process starts at the base station and it is then propagated hop by hop until covering all the network nodes. Node conditions are evaluated by using a fuzzy-logic-based mechanism, and only those nodes with better state (battery, number of hops to the sink) act as data forwarders (parent nodes).

The algorithm establishes minimum paths, in terms of energy wasting, from every node to the base station, which gathers data coming from all the network nodes. Roles are assigned to network nodes in order to balance the network load. These roles are leaf: nodes that sense the environment and send data to the base station, and master: nodes that as well as performing leaf node tasks, also forward data from other nodes to the base station.

In order to perform network organization through parent election and role assignment, NORIA stores neighboring data in a neighbor table, and uses the output of a fuzzy-logic system as a metric to make decisions.

NORIA defines four kinds of messages in order to organize the network and send collected data. These messages are: IPM (Information Propagation Message), which includes local information; RDM (Role Decision Message), which informs about selected role, MRM (Master Request Message), to request neighbor role changing, and DM (Data Message), which includes requested and/or forwarded data.

The algorithm consists of two stages: first, the algorithm organizes the network by creating the communication tree, which is routed at the base station, and by fixing as forwarder master nodes those with better conditions. The second stage is still being developed, and performs periodical verification, which includes, if necessary, role migration in order to provide load balancing and to avoid node overloading.

In the first stage of the algorithm, the communication tree is created and roles are assigned to nodes. The organization procedure starts when the base station sends an RDM. Nodes that receive this message send an IPM and start a timer. In this period of time, they wait for information messages from neighboring nodes. When the timer has expired, nodes perform parent election and make the role decision. An MRM is sent in case of the elected parent having a leaf role. Once the role has been decided, the nodes send an RDM to induce next-hop neighbors to start the organization phase.

The input variables to be considered in this experiments are: the number of hops to reach the base station, and the remaining energy. This parameters are an example of the full set of parameters which can be also included in the decision process (delivery probability, delay, signal strength... among others). The output variable represents the suitability of the node to be selected as parent node. Figure 2 shows fuzzy sets for input and output parameters. 


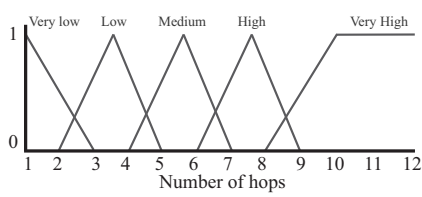

(a) Fuzzy Sets for Number of Hops.

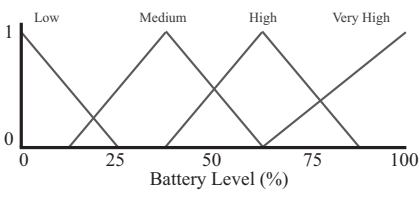

(b) Fuzzy Sets for Battery Level.

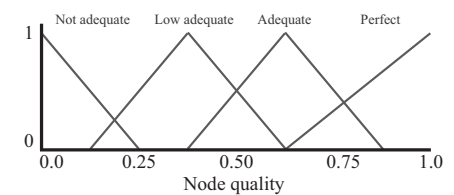

(c) Output Fuzzy Sets.

Fig. 2. Input Fuzzy sets.

Nodes will compare the output of the evaluation for each neighbor node to perform the parent selection process. Note that fuzzy input and output sets can be customized depending on the application and on the circumstances of each particular WSN. For example, in a network which needs real time data, it will be interesting the use of the end to end delay as a decision parameter.

For that example, the fuzzy rule base includes rules such us the following: if the Number of hops is Low and the Battery Level is High then the node quality is Adequate. Here, since we have 4 fuzzy sets for Battery level input and 5 for Number of hops input, we therefore have 20 rules, which are summarized in Table 1.

\section{Combining synchronized MAC with intelligent routing}

The integration of a synchronous MAC and an intelligent routing approach will make the network more accurate and efficient.Since SA-MAC uses just the number of hops of a neighbor to be elected as parent, integration with an efficient parent decision mechanism will be beneficial in terms of network performance and network load balance, avoiding forwarder-node overload and improving network lifetime.SA-MAC sends to NORIA the number of hops and the battery level of the neighbor node, and the fuzzy-logic-based decision process of NORIA provides the node quality value. Then, the node with the best quality value will be chosen as parent.

\subsection{Performance evaluation}

In order to evaluate the performance of the combined proposal, we have implemented both SA-MAC and NORIA in Omnet++ [14] simulator. We are going to consider circular network areas with constant node density. The network size varies from 50 to $250 \mathrm{~m}$, and the number of nodes varies from 21 to 489 . We have 
Table 1. Fuzzy rule base

\begin{tabular}{lll}
\hline N. of hops & Bat. Level & Node quality \\
\hline Very low & Low & Low adequate \\
Medium & High & Adequate \\
Very low & Medium & Low adequate \\
Medium & Very high & Adequate \\
Very low & High & Adequate \\
High & Low & Not adequate \\
Very low & Very High & Perfect \\
High & Medium & Low adequate \\
Low & Low & Not adequate \\
High & High & Low adequate \\
Low & Medium & Low adequate \\
High & Very high & Adequate \\
Low & High & Adequate \\
Very high & Low & Not adequate \\
Low & Very high & Perfect \\
Very high & Medium & Not adequate \\
Medium & Low & Not adequate \\
Very high & High & Low adequate \\
Medium & Medium & Low adequate \\
Very high & Very High & Adequate \\
\hline
\end{tabular}

used both grid and irregular topologies for the purpose of experimenting with different topologies and node deployments. The experiments have been executed 100 times in order to obtain reliable results.

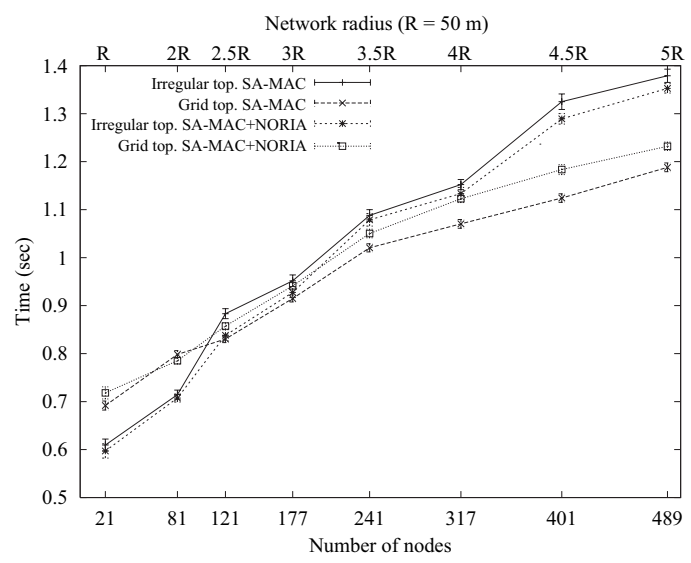

Fig. 3. Network set-up time. 


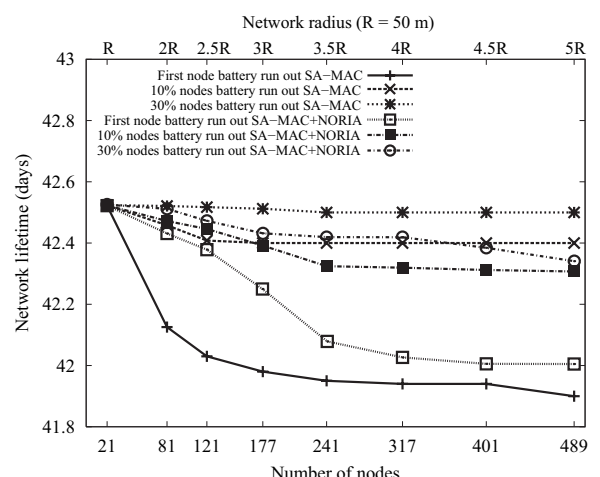

(a) Irregular topology.

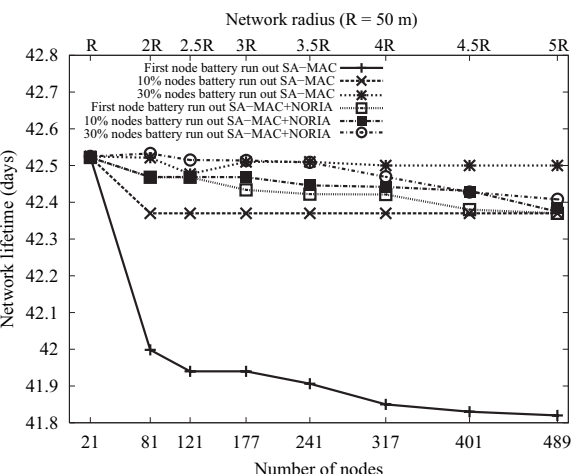

(b) Grid topology.

Fig. 4. Network lifetime.

Figure 3 shows that the addition of fuzzy logic only increments the set-up time when we are working with the grid topology, due to the fact that the nodes are frequently changing for a best parent, compared to the original SA-MAC way of working.

The lifetime of the network, in general terms, is improved. For both cases, grid and irregular, (see Fig. 4), the time in which the first node's battery run out is improved, and the gap between this event and $10 \%$ and $30 \%$ of the network lifetime is reduced, indicating that network workload has been balanced correctly in the continuous process of network re-configuration. What is noteworthy is the improvement on grid topology. Although the set-up time was slightly longer, this increase is not significant compared to the balance of workload among the nodes, and so the network lifetime significantly increased.

\section{Conclusions}

In this paper, we have integrated an existing TDMA-based MAC protocol with NORIA, an intelligent routing approach for WSNs.

Since the correct operation of a wireless sensor network requires that all nodes have to know who they have to send to, and at which moment, it is desirable that the network is both self-organized and synchronized. First of all, we have performed a deep study of previous work, and SA-MAC was selected to be integrated with a fuzzy-logic-based routing algorithm, NORIA.

Experimental results have shown that the combination of SA-MAC and NORIA reduces the depth of the communication tree, and that the network load has been balanced among nodes,thus increasing network lifetime.

These favorable results obtained in the simulator will be tested in a real application with the aim of confirm the improvements that the protocol integration provides. 


\section{References}

1. A. Boukerche, Algorithms and Protocols for wireless sensor networks, Wiley Ed, 2009.

2. D. Hristu-Varsakelis and W.S. Levine, Handbook of Networked and Embedded Control Systems. Springer-Verlag, 2004.

3. F. Royo, T. Olivares, and L. Orozco-Barbosa, A synchronous engine for wireless sensor networks, Telecommunication Systems 2009

4. A. M. Ortiz, T. Olivares, J. C. Castillo, L. Orozco, P. J. Marron and F. Royo. Intelligent Role-Based Routing for Dense Wireless Sensor Networks. WMNC 2010.

5. M. Macedo, A. Grilo, M. Nunes, Distributed latency-energy minimization and interference avoidance in TDMA wireless sensor networks. Computer Networks 2008.

6. W. Liao and H. Wang, An asynchronous MAC protocol for wireless sensor network, Journal of Network and Computer Applications 2008.

7. J. N. Al-Karaki, and A. E. Kamal. Routing Techniques in Wireless Sensor Networks: A survey. IEEE Wireless Communications 2004.

8. F. Dressler. A study of self-organization mechanisms in ad-hoc and sensor networks. Computer Communications Magazine 2008.

9. Q. Ren and Q. Liang, Energy-efficient Medium Access Control Protocols for Wireless Sensor Networks, Eurasip Journal on Wireless Communication and Networking 2006.

10. J. Wallace, D. Pesch, S. Rea and J. Irvine, Fuzzy Logic optimization of MAC parameters and Sleeping duty-cycles in wireless sensor networks, IEEE VTC 2005.

11. A. Bachir, M. Dohler, T. Watteyne and ,K. K. Leung, MAC essentials for wireless sensor networks, IEEE Communications Surveys and Tutorials 2010.

12. L. Reznik. Fuzzy Controllers. Newnes Publishing, Oxford, UK, 1997.

13. J.-S. R. Jang, C.-T. Sun, and E. Mizutani. Neuro-Fuzzy and Soft Computing: A Computational Approach to Learning and Machine Intelligence. Prentice Hall, 1997.

14. Omnet++ Simulator page, http://www.omnetpp.org/. 